Memorias del VII Encuentro Nacional de Experiencias en la Enseñanza de la Biología y la Educación Ambiental y II Congreso Nacional de Investigación en la Enseñanza de la Biología

\title{
LA ENSEÑANZA DE LA DIVERSIDAD FLORÍSTICA DEL CONTEXTO URBANO A PARTIR DE LA ESTRATEGIA DE PROYECTOS DE AULA
}

\section{TEACHING ABOUT FLORISTC DIVERSITY OF URBAN CONTEXT FROM THE CLASSROOM PROJECT STRATEGY}

\section{Rojas $\mathbf{M}^{1}$ \\ Rozo $\mathrm{T}^{2}$ \\ Beltrán $\mathrm{H}^{3}$}

\section{Resumen}

Con el fin de reconocer las problemáticas asociadas a la enseñanza de la diversidad florística en contextos urbanos, se realizó un trabajo de reconocimiento de la importancia ecológica y social de las especies presentes en el ITI Francisco José de Caldas (I.E.D). Lo anterior, hace de la escuela un escenario de acción, que acerca a los ciudadanos al reconocimiento y apropiacióndel recurso vegetal, haciendo frente a la pérdida de la identidad cultural y natural de los elementos nativos de la ciudad. Se desarrolló un trabajo bajo el modelo de investigación acción educativa. Se diseñaron e implementaron siete actividades orientadas hacia la formación investigativa y disciplinar que contribuyeron a la construcción de Proyectos de Aula desde el trabajo cooperativo y el uso de las Tecnologías de la Información y la Comunicación TIC's.Los estudiantes del ITI Francisco José de Caldas (I.E.D)diseñaronProyectos de Aula orientados hacia la construcción de herbarios, que buscaron la identificación de las especies exóticas y nativas presentes en la Institución y su asociación con las problemáticas ambientales. Además, se construyeron proyectos dirigidos hacia el reverdecimiento escolar como línea de intervención mediante la siembra de plantas nativas.Los Proyectos de Aula fueron una estrategia pertinente desde la enseñanza de las Ciencias Naturales y Educación Ambiental, que lograron la apropiación de la diversidad florística presente en el colegio, desde las líneas de trabajo propuestas que permitieron usar las herramientas de la Botánica para la sensibilización hacia su entorno natural.

Palabras clave: Diversidad florística, ciudad, Proyectos de Aula, Enseñanza de las Ciencias Naturales y Educación Ambiental.

\footnotetext{
1 Estudiante de Licenciatura en Biología. Grupo de Investigación en Calidad Ambiental (GICA) Universidad Distrital Francisco José de Caldas. thequen96@gmail.com

${ }^{2}$ Estudiante de Licenciatura en Biología.Grupo de Investigación en Calidad Ambiental (GICA) Universidad Distrital Francisco José de Caldas.etatisb@gmail.com

3 M. Sc. Pontificia Universidad Javeriana, Director del Grupo de Investigación en Calidad Ambiental
} (GICA) y Profesor Asociado Universidad Distrital Francisco José de Caldas.hebeltran@udistrital.edu.co 
Edición Extra-Ordinaria.ISSN 2027-1034 P. p 337 - 345

Memorias del VII Encuentro Nacional de Experiencias en la Enseñanza de la Biología y la Educación Ambiental y II Congreso Nacional de Investigación en la Enseñanza de la Biología

\section{Abstract}

In order to recognize the problems associate with the teaching of floristic diversity in urban context performed a reconnaissance work about social and ecological importance of the species present in the ITI Francisco José de Caldas (I.E.D). This makes the school a scene of action, citizens closer to the recognition and appropriation of vegetation, facing the loss of natural and cultural identity of the native elements of the city. This work was developed under the educational action research model. Were designed and implemented seven activities oriented research and discipline training that contributed to the construction of Classroom Projects from the cooperative work and the use of Information and Communication Technology (ICT). Students of ITI Francisco José de Caldas (I.E.D) designed Classroom Projects oriented towards building herbal, who sought the identification of exotic and native species present in the Institution and its association with environmental issues. Also,were performed projects aimed at schoolgreening, as a line of intervention by planting native plants. Classroom Projects were a relevant strategy from the teaching of Science and Environmental Education, who managed the appropriation of floristic diversity present in the school, from working lines proposals allowed to use of Botany tools for awareness towards their natural environment.

Key words: Floristic diversity, city, Classroom Projects, Teaching Science and Environmental Education.

\section{Introducción}

En contextos urbanos, la diversidad florística es importante por los servicios ambientales que presta y su valor simbólico, además, proveenbeneficios tangibles e intangibles de orden ambiental, estético, psicológico, paisajístico, recreativo, social y económico para la ciudad (Álvarezet al. 2004, Bolaños \& Moscoso, 2011; Mahecha et al., 2010).Lo anterior, refleja la importancia de conocer y apropiarse del recurso vegetal de la ciudad. En este sentido, la escuela se convierte en un escenario de acción frente a la pérdida de la identidad natural, a partir de estrategias relacionadas con la disciplina Botánica tales como la construcción de herbarios, la propagación de especies nativas y el reverdecimiento escolar. Igualmente, estas estrategias ofrecen la posibilidad para generar preguntas acerca de la realidad ambiental de los estudiantes y a su vez, permitir la apropiación de los elementos que conforman su entorno (Campos \& Niño, 2009; Córdoba \&Vargas, 2009).

Las zonas verdes en las instituciones educativas, pueden convertirse en nuevos escenarios de aprendizaje, mediante la estructuración de nuevas metodologías que permiten además del reconocimiento del potencial biológico, la posibilidad de vinculación de estudiantes y docentes como propositores de 
Edición Extra-Ordinaria.ISSN 2027-1034 P.p 337 - 345

Memorias del VII Encuentro Nacional de Experiencias en la Enseñanza de la Biología y la Educación Ambiental y II Congreso Nacional de Investigación en la Enseñanza de la Biología

alternativas de solución de las problemáticas que su contexto impone (Beltrán \& Giraldo, 2009; Cerda, 2001). Por lo anterior, surgen los Proyectos de Aula (PA) como estrategia de intervención pedagógica y didácticaque acercan a los estudiantes a los procesos de investigación, bajo la premisa de la enseñanza "enel contexto y para el contexto". En este sentido, permiten la participación crítica y reflexiva de los sujetos en su realidad natural (Beltrán \& Giraldo, 2009; Chaparro, 2003; Cerda, 2001; Jurado, 2003; Segura, 2003).El presente estudio tuvo como objetivo reconocer la diversidad florística presente en el Instituto Técnico Industrial Francisco José de Caldas (I.E.D) a partir de la estrategia de Proyectos de Aula en el área de Ciencias Naturales y Educación Ambiental.

\section{Metodología}

Este estudio se realizó durante cuatro meses con los estudiantes del Instituto Técnico Industrial Francisco José de Caldas (I.E.D), ubicado en la localidad de Engativá (Bogotá D.C.-Colombia). La propuesta se enmarcó dentro del modelo de investigación acción educativa (Latorre, 2005; McKernan, 1999; Rodríguezet al. 1996).

La construcción de los PA se realizó en tres fases a saber (Figura 1):

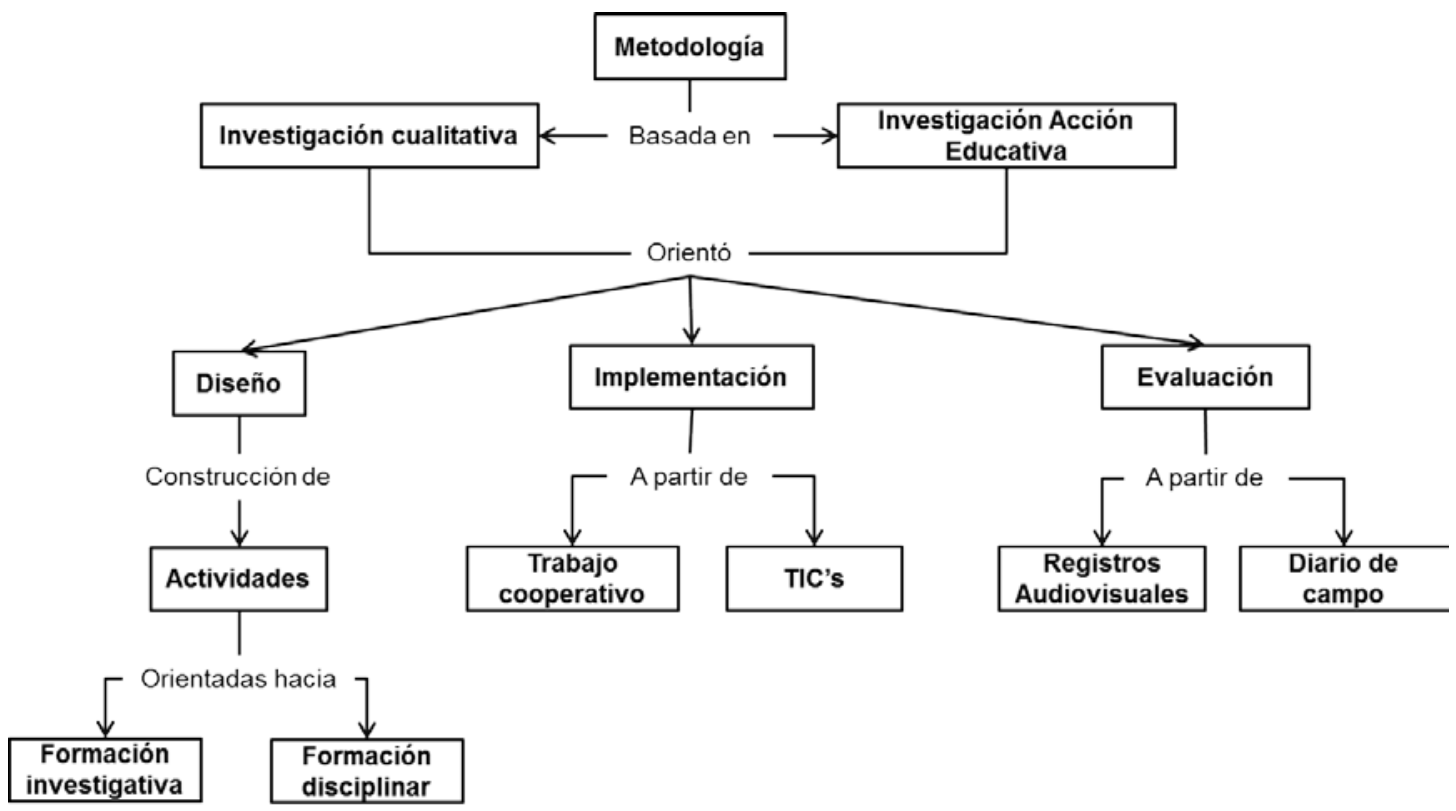

Figura 1. Esquema metodológico. 
Edición Extra-Ordinaria.ISSN 2027-1034 P. p 337-345

Memorias del VII Encuentro Nacional de Experiencias en la Enseñanza de la Biología y la Educación Ambiental y II Congreso Nacional de Investigación en la Enseñanza de la Biología

Diseño: Se construyeron actividades en Educación Ambiental conducentes al desarrollo de los PA por los estudiantes, desde las líneas de reverdecimiento escolar y construcción de herbarios, orientadas hacia la formación investigativa y disciplinar para fortalecer el trabajo en grupo, reconocer las realidades ambientales de su colegio, acercar a contextos investigativos e incorporar conceptos claves de la Botánica.

Implementación: Se utilizó la estrategia de trabajo cooperativo con la conformación de equipos para la formulación de los PA. Igualmente, se emplearon las Tecnologías de la Información y Comunicación TIC's (grupo en Facebook y googledocs.).

\section{Evaluación}

Se emplearon los diarios de campo docente y los registros video gráficos como instrumentos de reflexión docente de las percepciones acerca de los métodos y los resultados obtenidos durante la fase de implementación.

\section{Resultados Y Discusión}

Las actividades de formación en investigaciónpermitenla búsqueda, indagación, descubrimiento y asombro de la realidad desde el aprendizaje significativo (Cerda, 2001; Lacueva, 1998; Starico, 1996). Así mismo, las actividades dirigidas a la formación disciplinar,promueven el desarrollodel pensamiento científico para la construcción de conocimiento, reconocimiento y apropiación de conceptos propios del área de estudio (Campos \& Niño, 2009; MEN, 2006). Así, los estudiantes comprendieron las relaciones de interdependencia de su entorno y asumieron actitudes, valores y comportamientos que contribuyeron a la solución de las problemáticas ambientales relacionadas con la diversidad florística de la Institución (MEN, 1998; SINA, 2002; Torres, 2002).

En el presente estudio, se estructuraroncuatro PA dos en la línea de reverdecimiento escolar y dos en la línea de construcción herbarios a partir deltrabajo cooperativo, el grupo en Facebook y googledocs.(Figura 2). 
Edición Extra-Ordinaria.ISSN 2027-1034 P. p 337-345

Memorias del VII Encuentro Nacional de Experiencias en la Enseñanza de la Biología y la Educación Ambiental y II Congreso Nacional de Investigación en la Enseñanza de la Biología

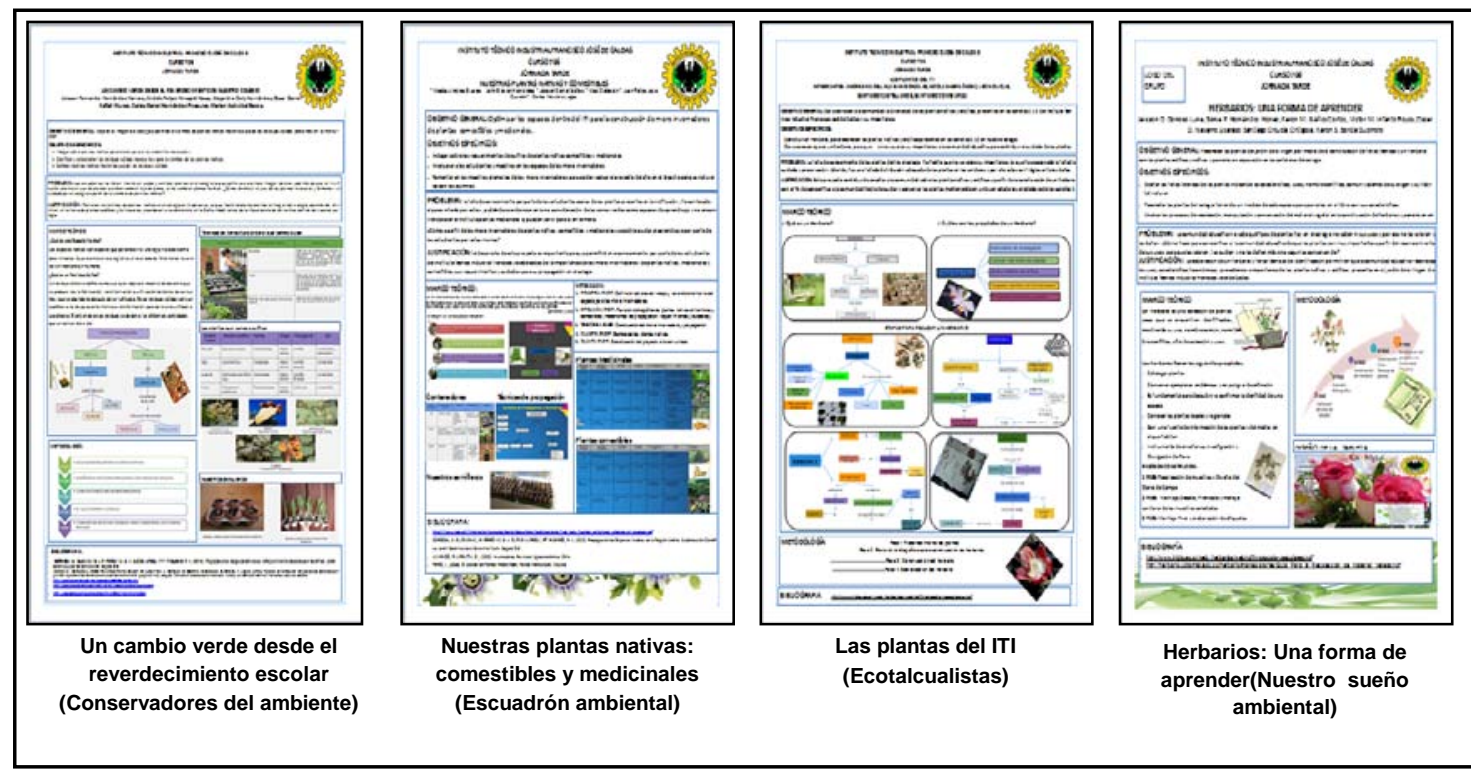

Figura 2.Poster de los Proyectos de Aula (PA) elaborados por los estudiantes.

Los grupos cooperativos de la línea de reverdecimiento escolar construyeron semilleros y contenedores de cultivo, elaboraron un jardín colgante y diseñaron quince fichas técnicas de las plantas nativas comestibles, medicinales y ornamentales que utilizaron en sus PA (Figura 3).

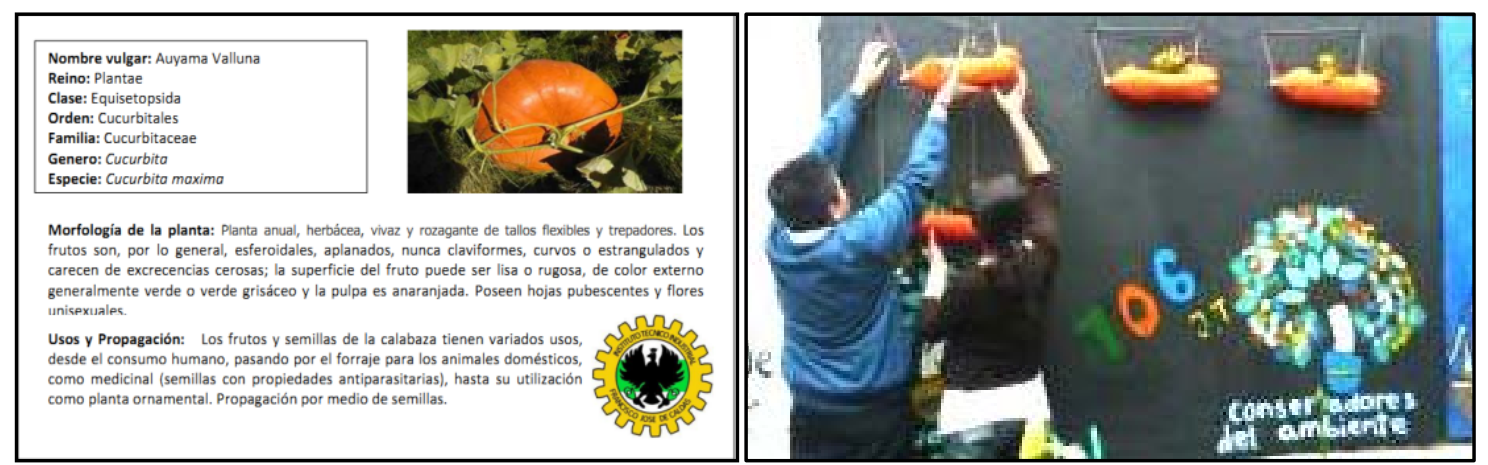

Figura 3.A la izquierda un ejemplo de las fichas técnicas y a la derecha el Jardín colgante elaborados por los estudiantes de la línea de reverdecimiento escolar.

En la línea de construcción de herbarios, los estudiantes identificaron y recolectaron 34 muestras de especies exóticas y 17 de especies nativas, las cuales fueron prensadas y secadas para organizarlas dentro de su pequeña colección. También, construyeron fichas informativas de las especies vegetales encontradas (Figura 4). 
Memorias del VII Encuentro Nacional de Experiencias en la Enseñanza de la Biología y la Educación Ambiental y II Congreso Nacional de Investigación en la Enseñanza de la Biología

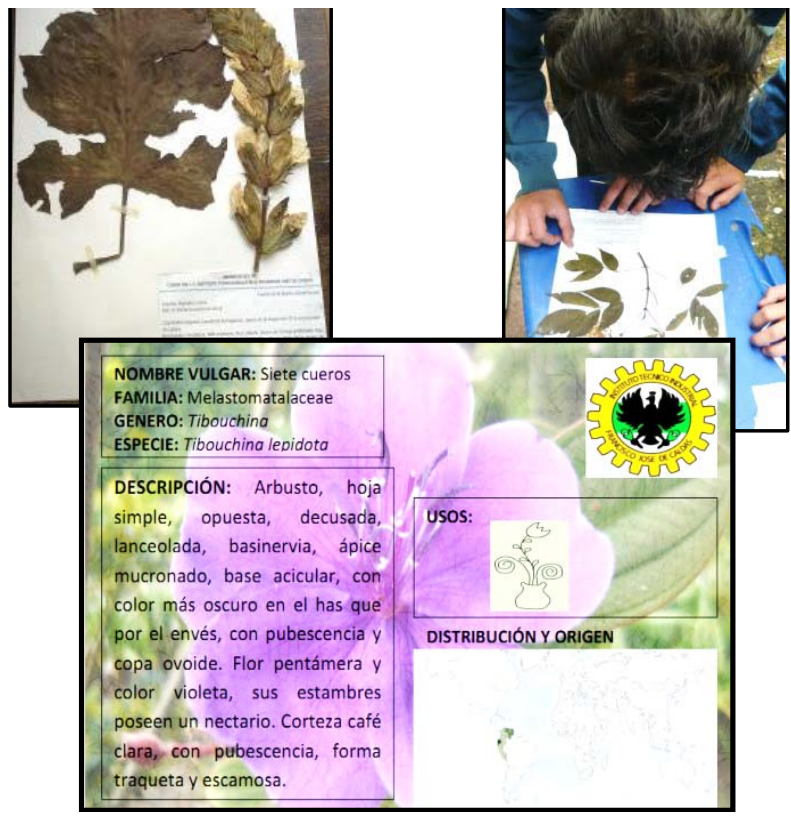

Figura 4.A la izquierda ejemplos del material vegetal procesado por los estudiantes y a la derecha la ficha informativa elaborada.

El trabajo cooperativo fue una estrategia que permitió a los estudiantes el desarrollo de habilidades y destrezas, el aprendizaje activo y autónomo y la toma de compromisos individuales y colectivos, facilitados con el uso de las TIC's que mejoraron los procesos de cooperación, respondiendo a las necesidades individuales de espacio y tiempo (Cerda, 2001; Guitertet al., 2007; MEN, 2006; Meso et al. 2012;Pujolás, 2009).

Los diarios de campo docentes arrojaron aspectos positivos y negativos de los métodos y resultados obtenidos en el aula: "...se mostró motivación y compromiso durante toda la fase de implementación de los proyectos...", "...se fomentó el sentido de pertenecía de los estudiantes hacia su trabajo...", "...el trabajo fuera del salón de clase presento dificultades en torno al dominio de grupo...", etc. Igualmente, los registros audiovisuales dieron a conocer las precepciones de los estudiantes acerca de las actividades realizadas: "...siempre fallamos en el trabajo en grupo, tenemos dificultades para reunirnos y comunicarnos para el trabajo...", "...googledocs., permitió que compartiéramos ideas y trabajáramos todos al mismo tiempo...", “...el trabajo elaborado sobre los herbarios me dio a conocer que lo que más me gusta es el tema de las plantas...", "...gracias a estos proyectos pudimos abrir nuevos espacios para aprender ciencias naturales...", “...con estos proyectos pudimos conocer plantas importantes para la ciudad como el nogal que es símbolo de Bogotá...", “...Este proyecto nos ayudó a comprender la problemática que estamos enfrentando con el ojo de poeta...” y “...empezamos a comprender la importancia de la siembra de plantas nativas para mantener nuestra biodiversidad...". 
Edición Extra-Ordinaria.ISSN 2027-1034 P. p 337 - 345

Memorias del VII Encuentro Nacional de Experiencias en la Enseñanza de la Biología y la Educación Ambiental y II Congreso Nacional de Investigación en la Enseñanza de la Biología

Tanto el diario de campo y las videograbaciones son mecanismos de facilitación parael desarrollo de una actitud científica e investigadora, la sensibilización ante la temática de interés "diversidad florística", el trabajo cooperativoy el papel del docente como mediador en los procesos dentro del aula, todas ellas características importantes dentro de la estrategia de PA (Chaparro, 2003; Jurado, 2003; Perilla \& Rodríguez, 2010; Segura, 2003).Además, problematizó la práctica educativa para su comprensión desde un proceso autorreflexivo que generóla reconceptualizaciónen la planificación e implementación de las actividades,valorando su efectividad (Elliot, 2005; Latorre, 2005; Mckernan, 1999)

\section{Conclusiones}

Los PA son una estrategia pertinente que favorecen la apropiación y sensibilización de los estudiantes hacia la diversidad florística de la ciudad desde la enseñanza de las Ciencias Naturales y Educación Ambiental.

Los PA fomentan la capacidad investigativa y creativa de docentes y estudiantes, vinculándolos a las realidades ambientales de su contexto y convirtiéndolos de agentes de acción y cambio.

Las Tecnología de la Información y Comunicación (TIC's) facilitan en los estudiantes el trabajo cooperativo, promueven la autonomía y generan sentido de pertenecía por losPA. Así mismo, permiten que los docentes asuman el rol de orientadores y facilitadores de los procesos.

\section{Lista de referencia}

Álvarez, G.; Tovar, G.; Bocanegra, F.; Chaparro, J.; Caicedo, G.; Rodríguez, D. \& Cardozo, L. (2004). Manual de silvicultura urbana para Bogotá. Bogotá, D. C.: Jardín Botánico José Celestino Mutis.

Beltrán, H., \& Giraldo, G. (2009). Elaboración de Proyectos de Aula. En H. Beltrán, L. Campos, C. Castaño, S. Córdoba, G. Giraldo, A. López, y otros, Talleres de formación: Expediciones Botánicas siglo XXI aprendiendo Ciencias con José Celestino Mutis (págs. 20-24). Bogotá, D.C.: Ministerio de Educación Nacional (MEN), Universidad Distrital Francisco José de Caldas.

Bolaños, T. \& Moscoso, A. (2011). Consideraciones y selección de especies vegetales parasu implementación en ecoenvolventesarquitectónicos: Una herramienta metodológica. Revista nodo. Vol. 5 (10). 5-20.

Campos, L. \& Niño, G. (2009). Elaboración de herbarios virtuales. . En H. Beltrán, L. Campos, C. Castaño, S. Córdoba, G. Giraldo, A. López, y otros, Talleres de formación: Expediciones Botánicas siglo XXI aprendiendo Ciencias 
Edición Extra-Ordinaria.ISSN 2027-1034 P. p 337 - 345

Memorias del VII Encuentro Nacional de Experiencias en la Enseñanza de la Biología y la Educación Ambiental y II Congreso Nacional de Investigación en la Enseñanza de la Biología

con José Celestino Mutis (págs. 20-24). Bogotá, D.C.: Ministerio de Educación Nacional (MEN), Universidad Distrital Francisco José de Caldas.

Cerda, H. (2001). El Proyecto de aula: El Aula como un Sistema de Investigación y Construcción de Conocimientos. Bogotá. D.C.: MAGISTERIO.

Chaparro, C. (2003). Proyectos de Aula vs Homogenización. Revista Internacional Magisterio. (2). 34- 36.

Córdoba, S., \& Vargas, M. (2009). Reverdecimiento Escolar. En H. Beltrán, L. Campos, C. Castaño, S. Cordoba, G. Fonseca, G. Giraldo, y otros, Talleres de formación docente: expediciones botánicas siglo XXI, aprendiendo ciencias con José Celestino Mutis (págs. 34-42). Bogotá, D.C.: Ministerio de Educación Nacional (MEN); Universidad Distrital Francisco José de Caldas.

Elliot, J. (2005). La investigación-acción en educación. 5a Edición. Madrid: Ediciones MORATA.

Guitert, M.; Romeu, T. \& Pérez, M. (2007). Competencias TIC y trabjo en equipo en entornos vistuales. Revista de Universidad y sociedad del conocimiento. Vol. 4 (1). 1-12.

Jurado, F. (2003). La educación por proyectos: una pedagogía para la conjetura.Revista Internacional Magisterio. (2), 18-22.

Lacueva, A. (1998). La enseñanza por proyectos ¿mito o realidad?.Revista Iberoamericana de Educación. (16). 165-187.

Latorre, A. (2005). La Investigación-Acción. En de Latorre, A. La InvestigaciónAcción: conocer y cambiar la práctica educativa (págs. 23-36). Barcelona: Graó.

Mahecha, E; Bocanegra, F.; Cadena, H.; Castro, J.; Chaparro, J.; Morales, G.; Quintero, M.; Sánchez, F., Tovar, G. \& Villota, L. (2010). Arbolado urbano de Bogotá: Identificación, Descripción y Bases para su manejo. Bogotá, D.C.: Alcaldía Mayor de Bogotá, D.C., Secretaría Distrital de Ambiente, SDA - Jardín Botánico de Bogotá José Celestino Mutis.

McKernan, J. (1999). Investigación-Acción y Curriculum. Madrid: Morata, S.L.

Meso, K.; Pérez, J. \&Mendiguren, T. (2012). Las redes sociales como herramienta para el aprendizaje colaborativo, presentación de un caso desde la UPV/EHU.

http://www.gabinetecomunicacionyeducacion.com/files/adjuntos/Las\%20redes 
Edición Extra-Ordinaria.ISSN 2027-1034 P. p 337 - 345

Memorias del VII Encuentro Nacional de Experiencias en la Enseñanza de la Biología y la Educación Ambiental y II Congreso Nacional de Investigación en la Enseñanza de la Biología

\%20sociales\%20como\%20herramientas\%20para\%20el\%20aprendizaje\%20col aborativo.\%20presentaci\%C3\%B3n\%20de\%20un\%20caso\%20desde\%20la\%2 OUPV_EHU.pdf

Ministerio de Educación Nacional (MEN). (1998). Ciencias Naturales y Educación Ambiental: lineamientos curriculares áreas obligatorias $y$ fundamentales. Bogotá D.C: Magisterio.

Ministerio de Educación Nacional (MEN) (ed). (2006). Estándares básicos de competencias en lenguaje, matemáticas, ciencias y ciudadanas. Bogotá, D.C.: Revolución educativa Colombia aprende.

Perilla, L., \& Rodriguez, E. (2010). Proyectos de Aula: Una estrategia Didáctica hacia el Desarrollo de Competencias Investigativas. EPISTEME, 1-9.

Pujolás, P. (2009). Introducción al aprendizaje cooperativo. Vich: Universidad de Vic

Rodriguez, G.; Gil, J., \& García, E. (1996). Metodología de la Investigación cualitativa. $2^{\mathrm{a}}$ edición. Macarena: EDICIONES ALJIBE.

Torres, M. (2002). Reflexión y Acción: Proyecto de Incorporación de la Dimensión Ambiental en zonas Rurales y pequeño Urbanas del País. Bogotá D.C: Ministerio de Educación Nacional (MEN); Ministerio de Ambiente y Desarrollo Sostenible.

Segura, D. (2003). Los proyectos de Aula más allá de una estrategia didáctica. Revista Intencional Magisterio. (2). 31- 33.

Sistema Nacional Ambiental (SINA). (2002). Política de Educación Ambiental. Bogotá, D.C.: Ministerio de Ambiente y Desarrollo Sostenible, Ministerio de Educación Nacional.

Starico, M. (1996). Los Proyectos de Aula hacia un aprendizaje significativo en la EGB. Argentina: Magisterio del Río de la Plata. 